\title{
Ultrasounds guided Dry needling: an effective technique for a long term relief of neck and shoulder pain
}

\author{
P. Fusco'1, V. de Paolis², F. De Sanctis², S. Di Carlo³, E. Di Martino³, F. Marinangeli², \\ C. Maggiani². \\ 1San Salvatore Academic Hospital of L'Aquila, Department of Anaesthesia and Intensive \\ Care Unit, L'Aquila, Italy. \\ 'University of L'Aquila, Department of Life- Healt and Environmental Sciences, L'Aquila, \\ Italy. \\ ${ }^{3}$ University of Chieti, Department of Anaesthesia- Resuscitation- Intensive and Pain Care, \\ L'Aquila, Italy.
}

Background and Aims:

Dry needling is a therapeutic technique in which thin monofilament needles are inserted into muscle eliciting a localized twitch response with the purpose of reducing pain associated with musculoskeletal disorder. These are often caused by the presence of Myofascial Trigger Points (MTrPs). Many RTCs comparing Dry needling with other treatments shows significant improvement in neck and shoulder pain, specifically in short and medium term. Performing DN under ultrasound guidance allows to identify MTrPs and to visualize local twitch response (LTR).

Methods:

A 31 years-old tattoo artist woman came to our observation complaining right neck and shoulder chronic pain. She referred a NRS pain score of 8 at rest raising 10 with movement, affecting her ability to work, McGill Pain Questionnaire=12(S6A3V1M2). She underwent several session of physiotherapy and taping and took NSAIDs as needed before our visit. We subjected her to 4 sessions of ultrasound guided dry needling: once every two weeks for two sessions and then once a month for the last two ones. We treated Upper trapezius, Sternocleidomastoid, Levator Scapulae, Semispinalis capitis, Splenius Capitis and Cervicis muscles.

Results:

We observed a marked reduction of local twitch response and NRS score, decreasing down to 0-1, McGill Pain Questionnaire=5 (S2A1V1M1). One year follow up confirmed absence of pain. The patient was able to return to daily and work activities without functional limitations caused by pain. Conclusions:

Ultrasounds guided dry needling treatment could be a safe and effective technique in the management of chronic neck and shoulder pain, even in the medium and long term.
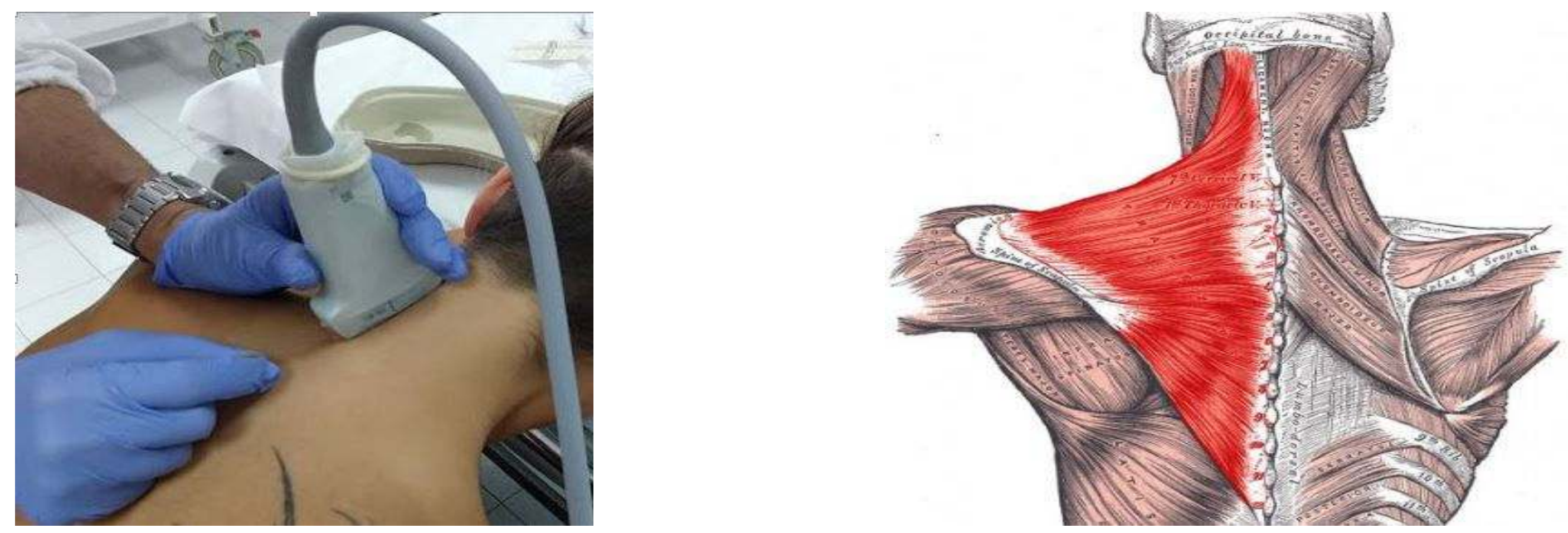